010 INSIGHTS INTO HYPERTENSIVE HEART DISEASE PHENOTYPES: SPECTRUM OF MYOCYTE, INTERSTITIAL AND VASCULAR CHANGES BY CARDIOVASCULAR MRI

1,2Jonathan CL Rodrigues, ${ }^{1,3}$ Antonio Matteo Amadu, 1,4Amardeep Ghosh Dastidar, ${ }^{1}$ Gergley Szantho, ${ }^{5}$ Laura EK Ratcliffe, ${ }^{4,5} \mathrm{Amy}$ E Burchell, ${ }^{2,5} \mathrm{Emma}$ C Hart, ${ }^{6}$ Mark CK Hamilton, ${ }^{4,5}$ Angus K Nightingale, 2,5 Julian FR Paton, ${ }^{6}$ Nathan E Manghat, ${ }^{1,4}$ Chiara Bucciarelli-Ducci. ${ }^{7}$ NIHR Bristol Cardiovascular Biomedical Research Unit, Cardiac Magnetic Resonance Department, Bristol Heart Institute, University Hospitals Bristol NHS Foundation Trust, Bristol; ${ }^{2}$ School of Physiology, Pharmacology and Neuroscience, Faculty of Biomedical Science, University of Bristol, Bristol; ${ }^{3}$ Department of Surgical, Microsurgical and Medical Sciences, Institute of Radiology, University of Sassari, Italy; ${ }^{4}$ Department of Cardiology, Bristol Royal Infirmary, University Hospitals Bristol NHS Foundation Trust, Bristol; ${ }^{5}$ CardioNomics Research Group, Clinical Research and Imaging Centre, Bristol Heart Institute, University Hospitals Bristol NHS Foundation Trust, Bristol; ${ }^{6}$ Department of Radiology, Bristol Royal Infirmary, University Hospitals Bristol NHS Foundation Trust, Bristol

\subsection{6/heartjn-2016-309680.10}

Introduction Hypertensive heart disease (HHD) can be classified into 4 left ventricular (LV) phenotypes: 1) normal, 2) concentric LV remodelling (LVr), 3) concentric LV hypertrophy (cLVH) and 4) eccentric LV hypertrophy (eLVH). The pathophysiology of the phenotypes is incompletely understood. We investigate extent of myocardial interstitial fibrosis and aortic impairment in HHD with cardiovascular magnetic resonance (CMR)

Methods 88 hypertensives $(49 \pm 14 y r s, 57 \%$ male, SBP: $167 \pm$ $30 \mathrm{mmHg}$, DBP: $96 \pm 14 \mathrm{mmHg}$ ) underwent CMR (1.5T) and were compared with 29 age- and sex-matched normotensive controls (47 \pm 14years, $59 \%$ male, SBP: $128 \pm 12 \mathrm{mmHg}$, DBP: $79 \pm 10 \mathrm{mmHg})$. Native T1 and extra-cellular volume fraction were measured. Circumferential myocardial strain was calculated by voxel-tracking. Aortic compliance was recorded.

Results At a structural level, increased LV mass in eccentric and concentric LVH resulted from: i) significantly increased myocardial cell volume (eLVH: $78 \pm 19 \mathrm{~g} / \mathrm{m}^{2}$ vs $\mathrm{cLVH}: 73 \pm 15 \mathrm{~g} / \mathrm{m}^{2}$ vs LVr: $55 \pm 9 \mathrm{~g} / \mathrm{m}^{2}$ respectively, $\left.\mathrm{P}<0.05\right)$ and ii) significantly increased interstitial volume (eLVH: $33 \pm 10 \mathrm{~g} / \mathrm{m}^{2}$ vs cLVH: 30 $\pm 10 \mathrm{~g} / \mathrm{m}^{2}$ vs $\mathrm{LVr}: 19 \pm 2 \mathrm{~g} / \mathrm{m}^{2}$ respectively, $\left.\mathrm{P}<0.05\right)$. Functionally, eccentric and concentric LVH were associated with significantly impaired circumferential strain (eLVH: $-12.8 \pm 4.6 \%$ vs cLVH: $-15.5 \pm 3.1 \%$ vs LVr: $-17.1 \pm 3.2 \%$ vs controls: $-17.4 \pm$ $2.6 \%$ respectively, $\mathrm{P}<0.05)$. Despite similar BP severity as $\mathrm{LVH}$ phenotypes, LV remodelling was associated with reduced aortic compliance but not associated with interstitial fibrosis or myocardial dysfunction relative to controls.

Conclusion Myocardial interstitial fibrosis is varies across HHD phenotypes with functional consequences. Eccentric LVH demonstrated the most interstitial fibrosis and systolic strain impairment. LV remodelling had normal myocardial, but abnormal aortic, function. Greater understanding of the pathophysiology of HHD phenotypes may help tailor future treatments.

\section{ROLE OF CORONARY COMPUTED TOMOGRAPHY ANGIOGRAPHY (CTA) IN CHEST PAIN EVALUATION IN FEMALES}

Hunain Shiwani, Christopher Saunderson, Mohammed Mannan Anwar, Mark Kon, Sudantha Bulugahapitiya. Bradford Royal Infirmary, Bradford, UK

\subsection{6/heartjnl-2016-309680.11}

Introduction Coronary computed tomography angiography (CTA) has a high negative predictive value for excluding significant coronary artery disease (CAD) with those with $<50 \%$ luminal stenosis not requiring further investigation. Those with
$>50 \%$ stenoses require further non-invasive or invasive evaluation. Current NICE guidelines recommend CT calcium scoring as first-line investigation in patients with chest pain and an estimated likelihood of CAD of 10-29\%. In females this includes those under 65 at low risk with atypical angina. The aim of this study was to evaluate coronary CTA as a single imaging modality to rule out significant CAD in females.

Methods We retrospectively assessed the coronary CTA findings of all female patients presenting with chest pain and undergoing assessment for possible underlying CAD at Bradford Royal Infirmary from January 2013 to December 2014. Patients were grouped according to age and this was assessed against the presence or absence of significant atheroma ( $>50 \%$ luminal stenosis). Results 462 female patients underwent Coronary CTA with a median age of 57 (range 31-82). Coronary CTA was able to rule out significant CAD in a high proportion of all age ranges but became less sensitive with advancing age. Those with a stenosis of greater than $50 \%$ required further evaluation. Exclusion of significant CAD varied from $90 \%$ for females aged $30-49$ to $87 \%$ in those aged 50-69 and remained as high as 78\% for those over 70 . Conclusion Coronary CTA provides an effective single modality evaluation for chest pain in females including those over 65 years of age.

\section{PRE RENAL TRANSPLANT CARDIAC RISK STRATIFICATION - CLINICAL RISK SCORE TO AID TRIAGE OF PATIENTS PRIOR TO NON-INVASIVE OR INVASIVE EVALUATION}

Christopher Saunderson, Owen Bebb, Sudantha Bulugahapitiya. Bradford Royal Infirmary, Bradford, UK

\subsection{6/heartjnl-2016-309680.12}

Introduction Stress myocardial perfusion imaging (MPI) is recommended to risk stratify patients undergoing renal transplantation. Patients with positive MPI studies are likely to need further invasive angiography. The aim of this study was to create a novel clinical risk score (CRS) to help triage patients to a single modality of non-invasive or invasive assessment prior to transplantation.

Method A review of the risk factor profile of 39 patients undergoing stress MPI prior to renal transplantation was undertaken. The CRS was developed as follows (one point if present): Age $>65$; BMI > 30; Smoking history; Hypertension; Diabetes; Vascular history. The CRS was applied to patients and compared with the outcome of the MPI.

Results 20 male and 19 female patients were identified. 4 patients were excluded (incomplete data). 5 patients had positive MPI studies. The characteristics of patients with positive and negative MPI is in Table 1 . The patient with a CRS score of zero had a negative MPI. 7.2\% (26/28) of patients with a CRS of 1-3 had a positive stress MPI compared with 50\% (3/6) in those with a CRS of 4 or greater. These patients required additional evaluation after MPI.

Conclusion

- Patients with CRS of zero may not need non-invasive assessment prior to renal transplant.

- Patients with a CRS of 1-3 are best assessed by non-invasive stress imaging initially.

- In patients with CRS of 4 and greater, invasive angiography may be more appropriate as the single investigative modality.

- Larger studies are needed to validate these findings. 